\title{
Could the static properties of nuclei be deduced from the dynamics of a single quark?
}

\author{
B. P. Kosyakovi ${ }^{1,2 *}$, E. Yu. Popov ${ }^{1}$, and M. A. Vronski1i1,3 \\ ${ }^{1}$ Russian Federal Nuclear Center-VNIIEF, Sarov, 607190 Nizhniŭ Novgorod Region, Russia; \\ ${ }^{2}$ Moscow Institute of Physics \& Technology, Dolgoprudny 141700 Moscow Region; Russia; \\ ${ }^{3}$ Sarov Institute of Physics \& Technology, Sarov, 607190 Nizhniĭ Novgorod Region, Russia. \\ E-mail address: kosyakov.boris@gmail.com
}

\begin{abstract}
We show that the static properties of a nucleus could arise from a single quark moving in a mean field generated by all other constituents of this nucleus. The resulting model provides a way for determining the nuclear sizes characteristic of the liquid drop model, and reasonably accurate values of magnetic moments of different nuclei with the aid of two parameters $\alpha_{s}$ and $\sigma$ appearing in the Cornell potential intended for use in quarkonium physics.
\end{abstract}

Keywords: effective theory, low energy QCD, static properties of nuclei

PACS numbers: 21.60.De, 21.10.Pc, 21.10 Ky, 14.40.Pq

Shortened version of the title:

Static properties of nuclei from the dynamics of a single quark

* Corresponding author 


\section{Introduction}

With the advent of quantum chromodynamics (QCD) a serious effort was mounted to understand nuclei in terms of quarks. Early in the development of this line of inquiry, a nucleus with mass number $\mathcal{A}$ was conceived as a system of $N=3 \mathcal{A}$ quarks moving in a large bag [1, [2]. However, the relationship between the number of quarks that are contained in a stable bag and its size $R$ is given 1 by $R \sim N^{1 / 4}$ which is contrary to the experimental nuclear data. Furthermore, the predicted nuclear magnetic moments significantly differ from their established values [4], [5]. We thus should proceed from another paradigm.

A direct way for clarifying the properties of a many-quark system is to use the Feynman path integral machinery whereby all degrees of freedom are integrated out, except for those of a single quark $Q$, so that this quark is affected by a mean field generated by all other constituents of the system. A systematic implementation of this calculation program is still a good distance in the future 2 . And yet the result of integrating out the remaining degrees of freedom can be neatly approximated if we make plausible assumptions about the mean field, and invoke the semiclassical approach for exploring the behavior of the quark $Q$. Is the semiclassical approach suitable to nuclear physics? It is pertinent to note that some properties of nuclei are typical of classical entities. For example, the observed size of a nucleus $R$ depends on mass number $\mathcal{A}$ as

$$
R=R_{0} \mathcal{A}^{1 / 3}
$$

which is peculiar to a classical liquid drop rather than a quantum object whose extension, characterized by its Compton wavelength, is inversely proportional to $\mathcal{A}$.

An attempt to develop these ideas showed considerable promise [7]. Some properties of nuclei are indeed amenable to this treatment. The nuclear force saturation (the property which became pressing in QCD-inspired approaches), and the nuclear scale characteristic of the liquid drop model, Eq. (11), are notable examples. We will show in the present paper that reasonably accurate values of magnetic moments for a rich variety of nuclei are also attainable in this analysis.

The dynamics of the quark $Q$, specified by the Dirac field $\psi$, is assumed to be encoded by the Lagrangian

$$
\mathcal{L}=\bar{\psi}\left[i \gamma^{\mu}\left(\partial_{\mu}+i g_{V} A_{\mu}\right)-m_{Q}\right] \psi+g_{S} \bar{\psi} \psi \Phi
$$

where $A_{\mu}=\left(A_{0}, \mathbf{A}\right)$ and $\Phi$ are respectively the Lorentz vector potential and Lorentz scalar potential of the mean field, $g_{V}$ and $g_{S}$ their associated couplings, and $m_{Q}$ the current-quark mass of the quark $Q$. The second interaction term in (2) is absent from the QCD Lagrangian because the scalar Yukawa coupling is contrary to asymptotic freedom. However, our concern is with the effective theory in the infrared region where the dynamics is anticipated to arrange itself into the form shown in Eq. (2).

\footnotetext{
${ }^{1}$ See, e. g., Ref. [3, Eq. (18.20).

${ }^{2}$ An effort to integrate out only gluon degrees of freedom was reasonably successful [6], but never progressed beyond small nuclei.
} 
The semiclassical treatment implies that the extremal path contribution dominates the Feynman path integral. This is the same as saying the wave function $\psi(x)$ of the quark $Q$ is a solution to the Dirac equation in the background $A_{\mu}(x)$ and $\Phi(x)$ because this equation results from the requirement that the action with the Lagrangian (2) be extremal. In fact, we restrict our attention to spherically symmetric static interactions, and assume that the contribution of the Lorentz vector potential to the mean field is given by $A_{0}$, that is, $\mathbf{A}=0$. What this means is a particle with reduced mass $m$ orbits the center of mass being driven by central potentials $A_{0}(r)$ and $\Phi(r)$. The assumption of the spherically symmetric interaction as applied to the case that the quark $Q$ moves in the mean field of an intricate form may seem awkward. However, the arguments in support of this assumption closely resemble those taken in the single-particle shell model of atomic nuclei in which the mean field exerting on every nucleon is given by a central potential because the nucleus in its ground state is approximately spherically symmetric, and the Pauli principle, acting through the already occupied orbitals, suppresses the role of long-range correlations [8]. We thus proceed from the Dirac Hamiltonian

$$
H=-i \alpha \cdot \nabla+U_{V}(r)+\beta\left[m+U_{S}(r)\right],
$$

where $U_{V}=g_{V} A_{0}$, and $U_{S}=g_{S} \Phi$.

It will be recalled that the Dirac equation can be safely regarded as a one-particle wave equation for interactions of a special kind. Following the conventional interpretation, positive energy states are attributed to a Dirac particle, while states of negative energy are attributed to its antiparticle. If there is a unitary transformation which diagonalizes the Dirac Hamiltonian with respect to positive and negative energies, then the wave functions of a Dirac particle of definite momentum have just two components, as it must for the usual quantum-mechanical interpretation of these wave functions to be adequate. Foldy and Wouthuysen [9] found a unitary transformation which diagonalizes the free Dirac Hamiltonian with respect to positive and negative energies. Case [10] obtained a closed form for this transformation in the presence of a time-independent magnetic field of arbitrary strength. The energy gap of the Dirac sea is not penetrated in the constant magnetic field because this field leaves the energy of the Dirac particle unchanged. Note also that the block-diagonalization is another way of stating that the system enjoys the property of supersymmetry [11]. The pseudoscalar coupling is a further fascinating candidate for the block-diagonalization of the Dirac Hamiltonian. Indeed, adding the term $g_{P} \bar{\psi} \gamma_{5} \psi \chi$ to (2), and setting $g_{V}=g_{S}=0$, we come to the Foldy-Wouthuysen picture. The systems with pseudoscalar couplings will be analyzed elsewhere.

Our prime interest in the present paper is with the systems which are governed by the Hamiltonian defined in (3) and subject to the spin- and pseudospin symmetry conditions (for an extended discussion of these phenomenological symmetries see Refs. [12] and [13]). The Foldy-Wouthuysen separation of positive- and negative-energy states is not attained in such systems. We will ascribe the spin symmetry condition to free hadrons, and the pseudospin symmetry condition to nuclei.

We take the Cornell potential [14]

$$
V_{\mathrm{C}}(r)=-\frac{\alpha_{s}}{r}+\sigma r
$$


as a phenomenological realization of both $U_{V}$ and $U_{S}$, and put $U_{V}=\frac{1}{2} V_{\mathrm{C}}$. Could the potential (44) be as much useful in nuclear physics as in the research of quarkonia? It will transpire in Sect. 3 that the pseudospin symmetry condition gives rise to a nucleus-sized cavity with singular boundary capable of keeping the quark $Q$ in this cavity from escaping whenever $U_{V}(r)$ grows indefinitely with $r$. We are thus free to vary rising potentials $U_{V}(r)$ and $U_{S}(r)$ in a wide range of their forms to attain the best fit to experiment. In the present paper, the form of $\left|U_{V}\right|$ and $\left|U_{S}\right|$ is fixed to be identical to that of $\frac{1}{2}\left|V_{\mathrm{C}}\right|$, and the values of $\alpha_{s}$ and $\sigma$ are borrowed from the description of quarkonia, - to examine what results in nuclear physics.

We are now in position to formulate our strategy. We assume that an effective theory to low energy QCD, which covers both free hadrons and nuclei, may arise when all degrees of freedom are integrated out, except for those of a single quark $Q$. To take the last step, that is, find the Feynman path integral over degrees of freedom of the quark $Q$, we would have to substitute this procedure with its semiclassical approximation. This leads us to the eigenvalue problem

$$
\left\{-i \alpha \cdot \nabla+U_{V}(r)+\beta\left[m+U_{S}(r)\right]\right\} \psi(\mathbf{r})=\varepsilon \psi(\mathbf{r}) .
$$

We look for solutions of this problem combined with the spin- and pseudospin symmetry conditions. We separate variables in the two-row Dirac eigenstates,

$$
\psi_{n_{r}, \ell, j, M}(\mathbf{r})=\left(\begin{array}{c}
f_{n_{r}, \ell}(r)\left[Y^{(\ell)}(\theta, \phi) \chi\right]_{M}^{(j)} \\
i g_{n_{r}, \ell, j}(r)\left[Y^{\left(\ell_{j}\right)}(\theta, \phi) \chi\right]_{M}^{(j)}
\end{array}\right) .
$$

Here, $f_{n_{r}, \ell}$ and $g_{n_{r}, \ell, j}$ are the radial amplitudes, $n_{r}, \ell, j$ are the radial, orbital, and total angular momentum quantum numbers, respectively, $\left[Y^{(\ell)}(\theta, \phi) \chi\right]_{M}^{(j)}$ stands for the coupled amplitude $\sum_{m, \mu} C_{m \mu M}^{\ell(1 / 2) j} Y^{(\ell)}(\theta, \phi) \chi_{\mu}, Y^{(\ell)}(\theta, \phi)$ is the spherical harmonic of order $\ell, \ell_{j}$ is given by $\ell_{\ell-1 / 2}=\ell-1$ and $\ell_{\ell+1 / 2}=\ell+1, \chi_{\mu}$ is the spin function. The operator $K=-\beta(\mathbf{S} \cdot \mathbf{L}+1)$ commutes with the spherically symmetric Dirac Hamiltonian. Thus, the Dirac eigenstates are the eigenstates of this operator with eigenvalues $\kappa= \pm\left(j+\frac{1}{2}\right)$, with '-' for aligned spin $\left(s_{1 / 2}, p_{3 / 2}\right.$, etc.), and ' + ' for unaligned spin $\left(p_{1 / 2}, d_{3 / 2}\right.$, etc. $)$, so that the quantum number $\kappa$ is sufficient to label the orbitals [15]. The radial part of Eq. (5) is

$$
\begin{gathered}
f^{\prime}+\frac{1+\kappa}{r} f-a g=0, \\
g^{\prime}+\frac{1-\kappa}{r} g+b f=0, \\
a(r)=\varepsilon+m+U_{S}(r)-U_{V}(r), \\
b(r)=\varepsilon-m-U_{S}(r)-U_{V}(r),
\end{gathered}
$$

where the prime stands for differentiation with respect to $r$.

We use (7) for expressing $g$ in terms of $f$ and substitute the result in (8) $)^{3}$. We eliminate the first derivative of $f$ from the resulting second-order differential equation

$$
f^{\prime \prime}+A f^{\prime}+B f=0,
$$

\footnotetext{
${ }^{3}$ Our concern is with $f$ because it is $f$ that survives in the nonrelativistic free-particle limit.
} 
in which

$$
A=-\frac{a^{\prime}}{a}+\frac{2}{r}, \quad B=a(1+\kappa)\left(\frac{1}{r a}\right)^{\prime}+a b+\frac{1-\kappa^{2}}{r^{2}},
$$

using the ansatz

$$
f=F \frac{\sqrt{a}}{r},
$$

to obtain the Schrödinger-like equation

$$
F^{\prime \prime}+k^{2} F=0,
$$

where

$$
k^{2}=\varepsilon^{2}-m^{2}-2 U(r ; \varepsilon)=-\frac{1}{2} A^{\prime}(r)-\frac{1}{4} A^{2}(r)+B(r) .
$$

Once all angular (orbital and spin) variables are thus eliminated, and the pertinent phenomenological condition on $U_{S}$ and $U_{V}$ is imposed, the function $U(r ; \varepsilon)$ defined in (15) acts as the effective potential.

The Schrödinger-like equation (14) is formally equivalent to the set of equations (7)-(8) [16]. However, the set of equations (7)-(8) is not diagonalizable, which seems invalidate the probabilistic interpretation of the two-row wave function of the Dirac particle $Q$. Meanwhile the particle $Q$ is not an ordinary quantum-mechanical particle because no quark can be isolated. From the conceptual point of view, the quark $Q$ defies its probing everywhere outside the region to which this quark is confined. We therefore will assume that $F$, the solution to the one-dimensional Schrödinger-like equation (14), is just the probability amplitude of the quark $Q$.

\section{Quarkonia}

Spin symmetry is inherent in free hadron states [17, [18. This symmetry occurs when $U_{S}=U_{V}$. With $V_{\mathrm{C}}=2 U_{V}$, Eqs. (15) and (12) can be solved to give the effective potential

$$
U(r ; \varepsilon)=\frac{1}{2}\left[\frac{\kappa(\kappa+1)}{r^{2}}+(\varepsilon+m)\left(-\frac{\alpha_{s}}{r}+\sigma r\right)\right] .
$$

In the nonrelativistic limit $\varepsilon \rightarrow m, U(r ; \varepsilon)$ becomes the sum of the centrifugal term and the Cornell potential, and hence, the results for the spectrum of quarkonia which were obtained through the use of the Schrödinger equation [19], [20] are reproduced in this procedure. It is then clear that spin-dependent effects appear as small corrections.

The procedure of looking for numerical solutions to Eqs. (7)-(10) with imposing the conditions $U_{S}=U_{V}=\frac{1}{2} V_{C}$ is outlined in Appendix. The agreement between the energy levels $\varepsilon_{n_{r}}$ obtained in this procedure and the masses of well established $c \bar{c}$ and $b \bar{b}$ states is within $\sim 1 \%$ for $\alpha_{s}=0.7, \sigma=0.14 \mathrm{GeV}^{2}, m_{c}=1.45 \mathrm{GeV}, m_{b}=4.92 \mathrm{GeV}$, see Table 1 which also lists the experimental data from [21].

A precise calculation of binary system energy levels should proceed from the use of the Bethe-Salpeter equation. However, both $c \bar{c}$ and $b \bar{b}$ are nonrelativistic systems, so that 
Table 1: Quarkonium masses

\begin{tabular}{|c|c|c|c|c|c|}
\hline$M_{c \bar{c}}$ & Calculation & Experiment & $M_{b \bar{b}}$ & Calculation & Experiment \\
\hline$J / \psi(1 S)$ & 3.084 & 3.097 & $\gamma(1 S)$ & 9.415 & 9.460 \\
\hline$\eta_{c}(1 S)$ & 3.084 & 2.980 & $\eta_{b}(1 S)$ & 9.415 & 9.398 \\
\hline$\psi(2 S)$ & 3.635 & 3.686 & $\gamma(2 S)$ & 10.04 & 10.023 \\
\hline$\eta_{c}(2 S)$ & 3.635 & 3.638 & $\eta_{b}(2 S)$ & 10.04 & 9.999 \\
\hline$\psi(3 S)$ & 4 & 4.030 & $\gamma(3 S)$ & 10.35 & 10.355 \\
\hline$\psi(4 S)$ & 4.3 & 4.421 & $\gamma(4 S)$ & 10.582 & 10.579 \\
\hline$\chi_{c 0}(1 P)$ & 3.511 & 3.414 & $\gamma(5 S)$ & 10.777 & 10.865 \\
\hline$\chi_{c 1}(1 P)$ & 3.511 & 3.510 & $\gamma(6 S)$ & 10.948 & 11.019 \\
\hline$\chi_{c 2}(1 P)$ & 3.511 & 3.556 & $\chi_{b 0}(1 P)$ & 9.98 & 9.859 \\
\hline$h_{c}(1 P)$ & 3.511 & 3.525 & $\chi_{b 1}(1 P)$ & 9.98 & 9.892 \\
\hline$\chi_{c 2}(2 P)$ & 3.897 & 3.929 & $\chi_{b 2}(1 P)$ & 9.98 & 9.912 \\
\hline$\psi(1 D)$ & 3.773 & 3.771 & $\chi_{b 0}(2 P)$ & 10.297 & 10.232 \\
\hline$\psi(2 D)$ & 4.095 & 4.153 & $\chi_{b 1}(2 P)$ & 10.297 & 10.255 \\
\hline & & & $\chi_{b 2}(2 P)$ & 10.297 & 10.268 \\
\hline & & & $1^{3} D_{2}$ & 10.222 & 10.161 \\
\hline
\end{tabular}

reasonably accurate results for the spectrum (which is experimentally measured to within $\sim 1 \%$ ) can be found by a nonrelativistic machinery, the Schrödinger equation governing the behavior of a particle with reduced mass $m=\frac{1}{2} m_{Q}$ in static confinement potentials, such as the Cornell potential [22, 23. The use of the Dirac equation for this purpose is as good as that of the Schrödinger equation.

\section{Nuclei}

The wavefunction classification stemming from pseudospin symmetry is important for both light and very heavy nuclei whose superdeformation appears already at low spin [24], [25]. The original observations that lead to the concept of "pseudospin symmetry" were quasi-degeneracies in spherical shell model orbitals with $\left(n_{r}, \ell, j=\ell+1 / 2\right)$ and $\left(n_{r}-1, \ell+2, j=\ell+3 / 2\right)$ where $n_{r}, \ell$, and $j$ are single-nucleon radial, orbital, and total orbital angular momentum quantum numbers, respectively. Pseudospin degeneracy in heavy nuclei derives from the fact that nucleons in a nucleus move in an attractive scalar, $-U_{S}$, and repulsive vector, $U_{V}$, mean fields, which are nearly equal in magnitude, $\left|U_{S}\right| \approx\left|U_{V}\right|$. This near equality of mean fields is likely a general feature of any relativistic model which fits nuclear binding energies [26].

One can go a step further and consider this condition as that arising from a quark $Q$ moving in a mean field generated by other quarks in the nucleus [27]. We adopt the condition $U_{S}=-U_{V}+C_{s}$ where $C_{s}$ is a constant. The Hamiltonian governing the quark 
$Q$ becomes

$$
H_{s}=\alpha \cdot \mathbf{p}+U_{V}(r)(1-\beta)+\beta\left(m+C_{s}\right) .
$$

We thus see that $m$ is shifted, $m \rightarrow m_{s}=m+C_{s}$. This shift may signal that the currentquark masses become the corresponding constituent-quark masses. In what follows $m_{s}$ will be regarded as the constituent-quark mass of the quark $Q$, and the label $s$ of $m_{s}$ will be omitted.

We put $V_{\mathrm{C}}=2 U_{V}$, and solve Eqs. (15) and (12) to give

$$
\begin{gathered}
U(r ; \varepsilon)=\frac{1}{2 r^{2}}\left\{\kappa(\kappa+1)+(\varepsilon-m)\left(-\frac{\alpha_{s}}{r}+\sigma r\right) r^{2}\right. \\
\left.+\frac{3\left(\alpha_{s}+\sigma r^{2}\right)^{2}}{4\left[\sigma r^{2}-(\varepsilon+m) r-\alpha_{s}\right]^{2}}+\frac{\alpha_{s}(\kappa+1)+\kappa \sigma r^{2}}{\sigma r^{2}-(\varepsilon+m) r-\alpha_{s}}\right\} .
\end{gathered}
$$

The terms in the first line of (18) closely resemble the respective terms involved in (16), except for changing the overall factor of the Cornell potential, but the terms of the second line dramatically change the situation. They are singular at $r=r_{\mathrm{sc}}$ which is the positive root of the equation $\sigma r^{2}-(\varepsilon+m) r-\alpha_{s}=0$,

$$
r_{\mathrm{sc}}=\frac{(\varepsilon+m)+\sqrt{(\varepsilon+m)^{2}+4 \sigma \alpha_{s}}}{2 \sigma} .
$$

To illustrate, the form of the effective potential (18) with some specific values of $m, \varepsilon, \alpha_{s}$, $\sigma$, and $\kappa$ is depicted in Fig. 1.

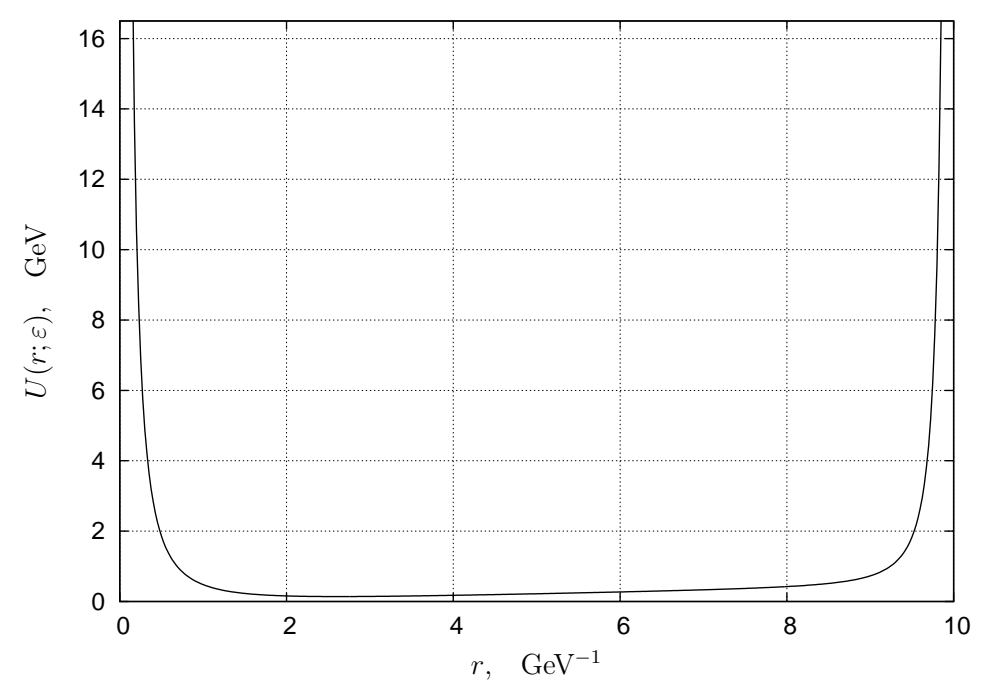

Figure 1: The effective potential (18) with the parameters $m=0.33 \mathrm{GeV}, \varepsilon=1 \mathrm{GeV}$, $\alpha_{s}=0.7, \sigma=0.14 \mathrm{GeV}^{2}, \kappa=1$

It is thus seen that the condition $U_{V}=-U_{S}=\frac{1}{2} V_{\mathrm{C}}$ vastly enhances the interaction between the quark $Q$ and the mean field to yield a spherical shell of radius $r_{\mathrm{sc}}$ on which 
$U(r ; \varepsilon)$ is infinite. The boundary of the spherical cavity of radius $r_{\mathrm{sc}}$ keeps the quark $Q$ in this cavity from escaping. It is well known [28 that the tunneling through a potential barrier of the form $\lambda\left(x-x_{0}\right)^{-2}$ with $\lambda \geq \frac{3}{4}$ is forbidden in one-dimensional quantum mechanics. This condition is fulfilled by Eq. (18)). Therefore, the boundary of the cavity sets up an impenetrable quantum-mechanical barrier.

A singular boundary arises whenever $U_{V}(r)$ grows indefinitely with $r$. This is because in going from Eqs. (7) and (8) to Eq. (14), we have to apply the factor $1 / a$ which is infinite when $a=0$. Such is not the case when we adopt the spin symmetry condition $U_{S}=U_{V}$ by which $a=\varepsilon+m$. In contrast, the pseudospin symmetry condition $U_{S}=-U_{V}$ implies that $a=\varepsilon+m-2 U_{V}$, and $a=0$ has a positive root provided that $U_{V}$ increases monotonically with $r$ beginning at $r=0$ where $U_{V}$ assumes a negative value. However, no singular boundary arises when $U_{V} \rightarrow U_{0}$ as $r \rightarrow \infty$, where $U_{0}$ is a constant which is less than $\frac{1}{2}(\varepsilon+m)$. This is the reason for the absence of confinement from systems with electromagnetic bindings.

It may be worth pointing out that the mathematical problem associated with Eq. (14) is not identical to the conventional eigenvalue problem for a one-dimensional Schrödinger Hamiltonian. Indeed, the effective potential $U(r ; \varepsilon)$, defined in (15)), depends nonlinearly on the parameter $\varepsilon$ which plays the role of an "eigenvalue" 4 . In contrast to the conventional eigenvalue problem where all energy levels are referred to a fixed effective potential $U(r)$, for every energy level $\varepsilon$ appearing in the present problem there is a unique potential configuration $U(r ; \varepsilon)$ exhibiting the spherical cavity of radius $r_{\mathrm{sc}}(\varepsilon)$ peculiar to just this 55. With this remark in mind it is little wonder that the behavior of the quark $Q$ in the state whose energy $\varepsilon$ is much greater than the constituent-quark mass $m$ may well be nonrelativistic because the singular interaction between this quark and the mean field of the nucleus converts the major portion of $\varepsilon$ to the mass content of the nucleus, expressed by the mass number $\mathcal{A}$, and only a tiny part of $\varepsilon$ is to be assigned to kinetic energy.

To verify that the effective potential $U(r ; \varepsilon)$ defined by Eq. (18) is indeed attributable to the description of nuclei, we solve numerically Eqs. (7) and (8) using the parameters $\alpha_{s}=0.7$ and $\sigma=0.1 \mathrm{GeV}^{2}$ (borrowed from the description of quarkonia), and taking $m$ to be $0.33 \mathrm{GeV}$. The procedure closely parallels that outlined in Appendix.

We find the energy levels $\varepsilon_{n_{r}}$ for $\kappa=-1,-2$ and the corresponding sizes of the cavities $r_{\mathrm{sc}}\left(n_{r}\right)$. To a good approximation the energy levels $\varepsilon_{n_{r}}$ turn out to be proportional to $\sqrt{n_{r}} 6$. By assuming that $n_{r}$ is proportional to $\mathcal{A}^{2 / 3}$, where $\mathcal{A}$ is the nucleon mass number,

\footnotetext{
${ }^{4}$ This raises the question of how to determine the radial quantum number $n_{r}$ in this context. Since $n_{r}$ equals the number of nodes of the radial part of the eigenfunction in the conventional eigenvalue problem, we take this fact as a way for determining $n_{r}$ by counting the nodes of $f_{n_{r}, \kappa}$ or $g_{n_{r}, \kappa}$.

${ }^{5}$ Note that the transition of the quark $Q$ from a given state specified by some $n_{r}$ to another state with lesser $n_{r}$ is forbidden because this transition would decrease the range of its localization (the impenetrable cavity would be smaller), with both energy levels being assumed to be definite, which is contrary to the Heisenberg's uncertainty principle.

${ }^{6}$ To offer a qualitative explanation of this relationship for large $n_{r}$, we recast Eqs. (7) and (8) as $G^{\prime \prime}+\left[\left(\kappa-\kappa^{2}\right) / r^{2}+\varepsilon\left(\varepsilon+m+\alpha_{c} / r-\sigma r\right)\right] G=0$, where $G=r g$. Since our concern is with large $r_{\mathrm{sc}}$, we may safely omit the terms proportional to $r^{-1}$ and $r^{-2}$. This gives the Airy equation. Regular solutions to this equation behave asymptotically as $\sin \left\{\frac{2}{3}(\varepsilon \sigma)^{1 / 2}[(\varepsilon+m) / \sigma-r]^{3 / 2}+\phi_{0}\right\}$, which shows that the number of radial nodes $n_{r}$ is estimated at $\left[\frac{2}{3}(\varepsilon \sigma)^{1 / 2} / \pi\right][(\varepsilon+m) / \sigma]^{3 / 2}$. Therefore, $\varepsilon_{n_{r}} \sim \sqrt{n_{r}}$.
} 
we come to the relationship $r_{\mathrm{sc}}=R_{0} \mathcal{A}^{1 / 3}$ with $R_{0} \approx 1 \mathrm{fm}$ for the chosen values of the $\alpha_{s}$ and $\sigma$, which is consistent with Eq. (1), for more details see Table 2.

Table 2: Calculated nuclei sizes

\begin{tabular}{|c|c|c|c|c|}
\hline Element & $\mathcal{A}$ & $n_{r}$ & $r_{\mathrm{sc}}(\mathrm{fm})$ & $R_{0}(\mathrm{fm})$ \\
\hline \multicolumn{5}{|c|}{ Neutron odd nuclei $(j=1 / 2, \varkappa=-1)$} \\
\hline${ }_{6}^{13} \mathrm{C}$ & 13 & 6 & 2.84 & 1.21 \\
\hline${ }_{8}^{15} \mathrm{O}$ & 15 & 6 & 2.84 & 1.15 \\
\hline${ }_{16}^{31} S$ & 31 & 10 & 3.6 & 1.14 \\
\hline${ }_{32}^{71} G e$ & 71 & 17 & 4.65 & 1.13 \\
\hline${ }_{32}^{\frac{102}{75} G e}$ & 75 & 18 & 4.8 & 1.14 \\
\hline${ }_{34}^{77} \mathrm{Se}$ & 77 & 18 & 4.8 & 1.13 \\
\hline${ }_{50}^{113} S n$ & 113 & 23 & 5.42 & 1.12 \\
\hline${ }_{52}^{119} \mathrm{Te}$ & 119 & 24 & 5.53 & 1.13 \\
\hline${ }_{68}^{169} \mathrm{Er}$ & 169 & 31 & 6.29 & 1.14 \\
\hline${ }_{70}^{171} Y b$ & 171 & 31 & 6.29 & 1.13 \\
\hline${ }_{80}^{181} \mathrm{Hg}$ & 181 & 32 & 6.4 & 1.13 \\
\hline $80 \mathrm{Hg}$ & 183 & 32 & 6.4 & 1.12 \\
\hline $80 \mathrm{Hg}$ & 185 & 32 & 6.4 & 1.12 \\
\hline${ }_{78}^{195} \mathrm{Pt}$ & 195 & 34 & 6.59 & 1.13 \\
\hline${ }_{78}^{197} \mathrm{Pt}$ & 197 & 34 & 6.59 & 1.13 \\
\hline${ }_{82}^{207} \mathrm{~Pb}$ & 207 & 35 & 6.69 & 1.13 \\
\hline${ }_{84}^{209} \mathrm{PO}$ & 209 & 35 & 6.69 & 1.13 \\
\hline \multicolumn{5}{|c|}{ Proton odd nuclei $(j=1 / 2, \varkappa=-1)$} \\
\hline${ }_{9}^{19} F$ & 19 & 7 & 3.03 & 1.14 \\
\hline${ }_{15}^{29} P$ & 29 & 9 & 3.43 & 1.12 \\
\hline $\begin{array}{l}31 \\
15\end{array}$ & 31 & 10 & 3.6 & 1.14 \\
\hline${ }_{55}^{125} \mathrm{Cs}$ & 125 & 25 & 5.65 & 1.13 \\
\hline${ }_{55}^{127} \mathrm{Cs}$ & 127 & 25 & 5.65 & 1.12 \\
\hline${ }_{55}^{129} \mathrm{Cs}$ & 129 & 26 & 5.77 & 1.12 \\
\hline $\begin{array}{l}1955 \mathrm{Tl} \\
81\end{array}$ & 195 & 34 & 6.58 & 1.13 \\
\hline${ }_{81}^{197} \mathrm{Tl}$ & 197 & 34 & 6.58 & 1.13 \\
\hline
\end{tabular}

\begin{tabular}{|c|c|c|c|c|}
\hline Element & $\mathcal{A}$ & $n_{r}$ & $r_{\text {sc }}(\mathrm{fm})$ & $R_{0}(\mathrm{fm})$ \\
\hline \multicolumn{4}{|c|}{ Neutron odd nuclei $(j=3 / 2, \varkappa=-2)$} \\
\hline${ }_{6}^{11} \mathrm{C}$ & 11 & 5 & 2.76 & 1.24 \\
\hline${ }_{16}^{33} \mathrm{~S}$ & 33 & 10 & 3.69 & 1.15 \\
\hline${ }_{16}^{35} \mathrm{~S}$ & 35 & 11 & 3.87 & 1.16 \\
\hline${ }_{16}^{35} \mathrm{Ar}$ & 35 & 11 & 3.87 & 1.16 \\
\hline${ }_{18}^{37} \mathrm{Ar}$ & 37 & 11 & 3.87 & 1.14 \\
\hline${ }_{18}^{39} \mathrm{Ca}$ & 39 & 12 & 4.02 & 1.18 \\
\hline${ }_{20}^{39} \mathrm{Ca}$ & 57 & 15 & 4.46 & 1.16 \\
\hline${ }_{28}^{57} \mathrm{Ni}$ & 57 & 1.13 \\
\hline${ }_{50}^{121} \mathrm{Sn}$ & 121 & 24 & 5.6 & 1.13 \\
\hline${ }_{52}^{129} \mathrm{Te}$ & 129 & 26 & 5.82 & 1.15 \\
\hline${ }_{54}^{131} \mathrm{Xe}$ & 131 & 26 & 5.82 & 1.15 \\
\hline${ }_{56}^{135} \mathrm{Ba}$ & 135 & 26 & 5.82 & 1.14 \\
\hline${ }_{56}^{137} \mathrm{Ba}$ & 137 & 27 & 5.93 & 1.15 \\
\hline${ }_{58}^{137} \mathrm{Ce}$ & 137 & 27 & 5.93 & 1.15 \\
\hline${ }_{58}^{139} \mathrm{Ce}$ & 139 & 27 & 5.93 & 1.15 \\
\hline${ }_{58}^{143} \mathrm{Ce}$ & 143 & 27 & 5.93 & 1.13 \\
\hline${ }_{74}^{187} \mathrm{~W}$ & 187 & 33 & 6.54 & 1.14 \\
\hline${ }_{76}^{189} \mathrm{Os}$ & 189 & 33 & 6.54 & 1.14 \\
\hline
\end{tabular}

The conjecture that $n_{r}$ equals the integral part of $\mathcal{A}^{2 / 3}$ has far-reaching implications, sending us in search of evidence for or against this conjecture. With this in mind, we compare the magnetic dipole of the quark $Q$ and that of the nucleus in which this quark is incorporated. Note that the magnetic moment of a nucleon in the states with isospin $I$ and the total angular momentum $j=\ell+\frac{1}{2}$ and $j=\ell+\frac{3}{2}$ is given [29], respectively, by $\mu_{j, I}=-\frac{e \gamma_{I}\left(j+\frac{1}{2}\right)}{2(j+1)} \int_{0}^{\infty} f_{n_{r}-1, \ell+1, j, I} g_{n_{r}-1, \ell+1, I} r^{3} d r+\mu_{A, I}\left(1-\frac{2 j+1}{j+1} \int_{0}^{\infty} g_{n_{r}-1, \ell+1, I}^{2} r^{2} d r\right)$, 
and

$\mu_{j, I}=\frac{e \gamma_{I}\left(j+\frac{1}{2}\right)}{2(j+1)} \int_{0}^{\infty} f_{n_{r}-1, \ell+1, j, I} g_{n_{r}-1, \ell+1, I} r^{3} d r-\frac{\mu_{A, I}}{j+1}\left[j-(2 j+1) \int_{0}^{\infty} g_{n_{r}-1, \ell+1, I}^{2} r^{2} d r\right]$.

Here, $\gamma_{I}$ is the orbital gyromagnetic ratio, $\mu_{A, I}$ is the anomalous magnetic moment, $\mu_{A,-\frac{1}{2}}=-1.913 \mu_{\mathrm{B}}, \mu_{A, \frac{1}{2}}=1.793 \mu_{\mathrm{B}}$, where $\mu_{\mathrm{B}}=e / 2 M$ is the nucleon magneton. We reiterate mutatis mutandis the arguments of Ref. [30] to conclude that (20) and (21), in which the infinite limits of integration are replaced by $r_{\mathrm{sc}}$, are well suited for the magnetic moment of neutron-odd nuclei with $j=\frac{1}{2}$ and $j=\frac{3}{2}$ to be represented by the magnetic moment of a constituent $u$ quark contained in these nuclei, with the understanding that $n_{r}$ is associated with $\left[\mathcal{A}^{2 / 3}\right]$, where the square brackets denote the integral part of the quantity enclosed in them. We determine the quark wave functions $f_{n_{r}, j}$ and $g_{n_{r}, j}$ for $\alpha_{s}=0.7, \sigma=0.14 \mathrm{GeV}^{2}, m=0.33 \mathrm{GeV}$. As to the anomalous magnetic moments of quarks, the present notion of their values is far from complete. Among suggested values of $\mu_{A}$ for $u$ quarks [31, 32, we adopt to test $\mu_{A}=0.15 \mu_{\mathrm{B}}$, and $0.2 \mu_{\mathrm{B}}$.

Fig. 2(a,b) shows that the magnetic moments of neutron-odd nuclei with $j=\frac{1}{2}$ and $j=\frac{3}{2}$ [33] agree with the calculated magnetic moments of a $u$ quark involved in those nuclei to an accuracy of $\sim 20 \%$. The exceptions are ${ }_{50}^{113} \mathrm{Sn}$ and ${ }_{52}^{119} \mathrm{Te}$ whose magnetic moments are consistent with the results of our calculation to within $50 \div 90 \%$. ${ }_{50}^{113} \mathrm{Sn}$ and ${ }_{52}^{119} \mathrm{Te}$ have respectively 50 and 52 protons. 50 is a magic number whereas 52 is not. Other neutron-odd nuclei with magic numbers of protons ${ }_{8}^{15} \mathrm{O}$ and ${ }_{82}^{207} \mathrm{~Pb}$ in the state with $j=\frac{1}{2}$, and ${ }_{20}^{39} \mathrm{Ca},{ }_{28}^{57} \mathrm{Ni}$ and ${ }_{50}^{121} \mathrm{Sn}$ in the state with $j=\frac{3}{2}$ ) do not exhibit this discrepancy.

Similar calculations can be done for proton-odd nuclei with $j=\frac{1}{2}$, that is, with the use of Eq. (21). It is seen from Fig. 2(c) that the accuracy of $\sim 20 \%$ between the results of our calculations and the data for the magnetic moments of such nuclei [34] can be attained if we turn to the behavior of a $u$ quark. Although it would be more physically reasonable to invoke the calculated magnetic moments of a $d$ quark for comparing them with those of proton-odd nuclei, the use of a $u$ quark for this purpose results in a better fit. This fact is rather puzzling and should be illuminated in the subsequent studies.

Since a single proton may be regarded as both a free hadron and the lightest nucleus, ${ }_{1}^{1} \mathrm{H}$, that is, a system subject to both spin- and pseudospin symmetry conditions, which are in conflict with each other, our argument is unsuitable for it. In this connection, the idea of spherical singular cavity is to be applied to light nuclei (which fall within a transition region between the spin- and pseudospin symmetry coverages) with care. The derivation of the properties of light nuclei in the framework of an effective theory to low-energy QCD is an intricate problem in itself. The severity of the problem was recently manifested in Ref. [35] by giving theoretical evidence that six-quark color-neutral configurations compatible with the physical states of dibaryons cannot form stable bound states.

\section{Conclusion}

Let us summarize the main results of this paper. We consider the behavior of a single quark $Q$ contained in some nucleus, assuming that all remaining degrees of freedom of 

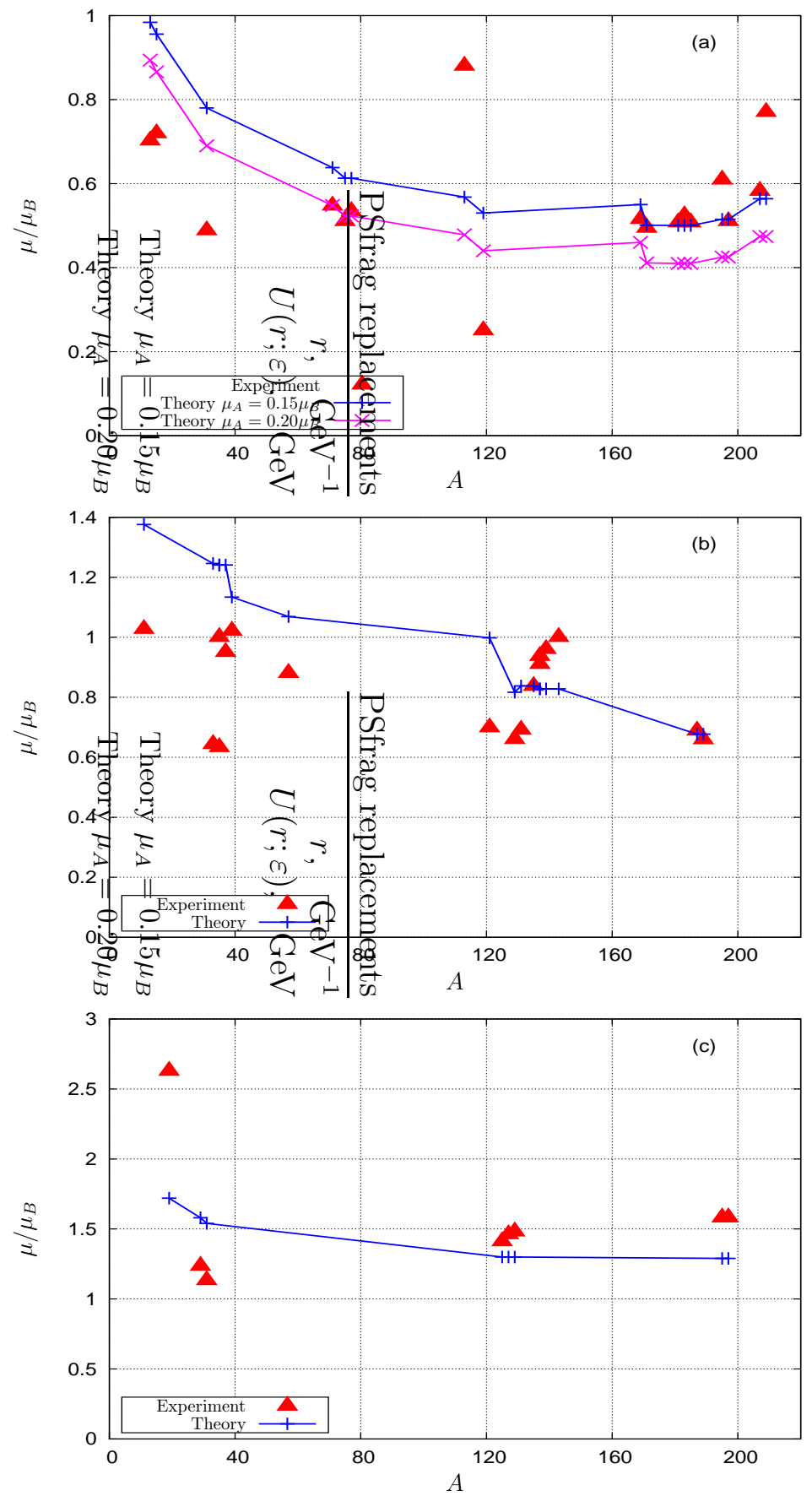

Figure 2: Comparison of the calculated magnetic moments with their experimental values for (a) neutron-odd $(j=1 / 2)$, (b) neutron-odd $(j=3 / 2)$, (c) proton-odd $(j=1 / 2)$ nuclei

this nucleus were already integrated out, and the result of this integration yields a mean field which exerts on the quark $Q$. We anticipate that the quark $Q$ is governed by the Lagrangian (2). We invoke the semiclassical treatment, which implies that the extremal path contribution dominates the Feynman path integral. Thus, the wave function of the 
quark $Q$ bears on a solution to the Dirac equation. The quark $Q$ is affected by the Lorentz vector potential $A_{\mu}(x)$ and Lorentz scalar potential $\Phi(x)$ of the mean field. We restrict our attention to spherically symmetric static interactions between the quark $Q$ and the mean field, and assume that the contribution of $A_{\mu}$ to the mean field is given by $A_{0}$. The pseudospin symmetry condition is imposed on $U_{V}=g_{V} A_{0}$ and $U_{S}=g_{S} \Phi$, namely $U_{S}=-U_{V}+C_{s}$. We take the Cornell potential (4) as a phenomenological realization of both $U_{V}$ and $U_{S}$, and put $U_{V}=\frac{1}{2} V_{\mathrm{C}}$. This allows understanding of the fact that the interaction between the quark $Q$ and this mean field yields a spherical cavity of radius $r_{\mathrm{sc}}$ with the boundary where the effective potential $U(r ; \varepsilon)$ is infinite. This keeps the quark $Q$ in this cavity from escaping. We solve numerically Eq. (14) with the parameters of the Cornell potential borrowed from the description of quarkonia, and obtain the energy levels $\varepsilon$ for $\kappa=-1,-2$ and the corresponding sizes of the cavities $r_{\mathrm{sc}}(\varepsilon)$. The energy levels $\varepsilon_{n_{r}}$ turn out to be proportional to $\sqrt{n_{r}}$, where $n_{r}$ is the radial quantum number determined by counting the nodes of the radial amplitude $f_{n_{r}, \kappa}$. We assume that $n_{r}$ equals the integral part of $\mathcal{A}^{2 / 3}$, where $\mathcal{A}$ is the nucleon mass number. This gives the relationship $r_{\mathrm{sc}}=R_{0} \mathcal{A}^{1 / 3}$, which is characteristic of the liquid drop model. To verify that the assumption $n_{r}=\left[\mathcal{A}^{2 / 3}\right]$ is consistent with the experimental data, we compare the magnetic dipole of the quark $Q$ and that of the nucleus in which this quark is incorporated. The agreement between the calculated and observed values of $\mu / \mu_{\mathrm{B}}$ is for the most part within $\sim 20 \%$ which is better than expected when taken into account that the picture in which a single quark moving in a static spherically symmetric mean field applies to a rich variety of nuclei whose dynamical contents are highly tangled.

\section{Acknowledgements}

We thank S. Brodsky, M. Chaichian, E. Epelbaum, J. Ginocchio, T. Goldman, and K. Yazaki for fruitful discussions. Critical remarks of an anonymous referee were of benefit to further clarification of the present text.

\section{Appendix A}

We outline here the procedure of looking for numerical solutions to Eqs. (77)-(10) with imposing the conditions $U_{S}=U_{V}=\frac{1}{2} V_{\mathrm{C}}$. Since our interest is with solutions that are regular at $r=0$, we use the ansätze $f(r)=u(r) r^{|\kappa|-1}$ and $g(r)=v(r) r^{|\kappa|-1}$. The functions $u$ and $v$ satisfy the following integral equations

$$
\begin{gathered}
u(r)=\frac{\kappa-|\kappa|}{\alpha_{s}}+(\varepsilon+m) \int_{0}^{r} d s\left[\theta(-\kappa)+\theta(\kappa)\left(\frac{s}{r}\right)^{2|\kappa|}\right] v(s), \\
v(r)=1+\int_{0}^{r} d s\left\{(\sigma s-\varepsilon+m)\left[\theta(\kappa)+\theta(-\kappa)\left(\frac{s}{r}\right)^{2|\kappa|}\right] u(s)+\frac{\alpha_{s}(\varepsilon+m)}{2|\kappa|}\left[\left(\frac{s}{r}\right)^{2|\kappa|}-1\right] v(s)\right\},
\end{gathered}
$$


where $\theta(\kappa)$ is the Heaviside step function. We write $u$ and $v$ as the Liouville-Neumann series,

$$
u=\sum_{k=0}^{\infty} u_{k}, \quad v=\sum_{k=0}^{\infty} v_{k},
$$

where $u_{0}=(\kappa-|\kappa|) / \alpha_{s}, v_{0}=1$,

$$
\begin{gathered}
u_{k+1}=\Phi_{12} v_{k}, \quad v_{k+1}=\Phi_{21} u_{k}+\Phi_{22} v_{k}, \quad k \geq 0, \\
\Phi_{12} v(r)=(\varepsilon+m) \int_{0}^{r} d s\left[\theta(-\kappa)+\theta(\kappa)\left(\frac{s}{r}\right)^{2|\kappa|}\right] v(s), \\
\Phi_{21} u(r)=\int_{0}^{r} d s(\sigma s-\varepsilon+m)\left[\theta(\kappa)+\theta(-\kappa)\left(\frac{s}{r}\right)^{2|\kappa|}\right] u(s), \\
\Phi_{22} v(r)=\frac{\alpha_{s}(\varepsilon+m)}{2|\kappa|} \int_{0}^{r} d s\left[\left(\frac{s}{r}\right)^{2|\kappa|}-1\right] v(s) .
\end{gathered}
$$

Every term of these series is a polynomial. The coefficient of a given monomial can be found recursively taking into account that

$$
\begin{gathered}
\Phi_{12}\left(r^{k}\right)=(\varepsilon+m)\left[\frac{\theta(-\kappa)}{k+1}+\frac{\theta(\kappa)}{k+1+2|\kappa|}\right] r^{k+1} \\
\Phi_{21}\left(r^{k}\right)=\sigma\left[\frac{\theta(\kappa)}{k+2}+\frac{\theta(-\kappa)}{k+2+2|\kappa|}\right] r^{k+2}-(\varepsilon-m)\left[\frac{\theta(\kappa)}{k+1}+\frac{\theta(-\kappa)}{k+1+2|\kappa|}\right] r^{k+1} \\
\Phi_{22}\left(r^{k}\right)=-\frac{\alpha_{s}(\varepsilon+m)}{(k+1)(k+1+2|\kappa|)} r^{k+1}
\end{gathered}
$$

\section{References}

[1] K. Bleuler et al., Nuclear shell structure in the light of quark theory Z. Naturforsch. 38a, 705 (1983).

[2] H. R. Petry et al., An application of QCD in nuclear structure. Phys. Lett. B 159, $363(1985)$.

[3] F. E. Close, An Introduction to Quarks and Partons (Academic, New York, 1979).

[4] A. Arima, K. Yazaki, H. Bohr, A quark shell model calculation of nuclear magnetic moments. Phys. Lett. B 183, 131 (1987).

[5] I. Talmi, The nuclear shell model - of nucleons or quarks? Phys. Lett. B 205, 140 (1988).

[6] K. Maltman, G. J. Stephenson Jr., T. Goldman, A relativistic quark model of nuclear substructure in the $A=3$ system. Phys. Lett. B 324, 1 (1994). 
[7] B. P. Kosyakov, E. Yu. Popov, M. A. Vronskiŭ, The bag and the string: Are they opposed? Phys. Lett. B 744, 28 (2015).

[8] J. D. Walecka, Theoretical Nuclear and Subnuclear Physics, 2nd ed. (World Scientific, Singapore, 2004). Ch. 5.

[9] L. L. Foldy, S. A. Wouthuysen, On the Dirac theory of spin- $\frac{1}{2}$ particles and its nonrelativistic limit, Phys. Rev. 78, 29 (1950).

[10] K. M. Case, Some generalizations of the Foldy-Wouthuysen transformation, Phys. Rev. 95, 1323 (1954).

[11] R. P. M. Romero, M. Moreno, A. Zentella, Supersymmetric properties and stability of the Dirac sea. Phys. Rev. D 43, 2036 (1991).

[12] J. N. Ginocchio, Relativistic symmetries in nuclei and hadrons. Phys. Rep. 414, 165 (2005).

[13] H. Liang, J. Meng, S.-G. Zhou, Hidden pseudospin and spin symmmetries and their origins in atomic nuclei. Phys. Rep. 570, 1 (2015) [arXiv: 1411.6774].

[14] E. Eichten et al., Spectrum of charmed quark-antiquark bound states. Phys. Rev. Lett. 34 (1975) 369.

[15] J. N. Ginocchio, Relativistic symmetries in nuclei and hadrons. Phys. Rep. 414, 165 (2005).

[16] H. Liang, J. Meng, S.-G. Zhou, Hidden pseudospin and spin symmmetries and their origins in atomic nuclei. Phys. Rep. 570, 1 (2015) [arXiv: 1411.6774].

[17] P. R. Page, T. Goldman, J. N. Ginocchio, Relativistic symmetry suppresses quark spin-orbit splitting. Phys. Rev. Lett. 86, 204 (2001) [arXiv: hep/ph/0002094].

[18] J. N. Ginocchio, Relativistic symmetries in nuclei and hadrons. Phys. Rep. 414, 165 (2005).

[19] E. Eichten et al., Charmonium: Comparison with experiment. Phys. Rev. D 21, 203 (1980).

[20] T. Barnes, S. Godfrey, E. S. Swanson, Higher charmonia. Phys. Rev. D 72, 054026 (2005) [arXiv: hep-ph/0505002].

[21] K. A. Olive et al., (Particle Data Group), Review of particle physics. Chin. Phys. C 38, 090001 (2014).

[22] E. Eichten et al., Charmonium: Comparison with experiment. Phys. Rev. D 21, 203 (1980).

[23] T. Barnes, S. Godfrey, E. S. Swanson, Higher charmonia. Phys. Rev. D 72, 054026 (2005) [arXiv: hep-ph/0505002]. 
[24] J. N. Ginocchio, Relativistic symmetries in nuclei and hadrons. Phys. Rep. 414, 165 (2005).

[25] H. Liang, J. Meng, S.-G. Zhou, Hidden pseudospin and spin symmmetries and their origins in atomic nuclei. Phys. Rep. 570, 1 (2015) [arXiv: 1411.6774].

[26] J. N. Ginocchio, Pseudo-spin as a relativistic symmetry. Phys. Rev. Lett. 78, 436 (1997) [arXiv: nucl-th/9611044].

[27] B. P. Kosyakov, E. Yu. Popov, M. A. Vronskilu, The bag and the string: Are they opposed? Phys. Lett. B 744, 28 (2015).

[28] J. Dittrich, P. Exner, Tunneling through a singular potential barrier. J. Math. Phys. 26, 2000 (1985).

[29] J. N. Ginocchio, Implication of pseudospin symmetry on relativistic magnetic properties and Gamow-Teller transitions in nuclei. Phys. Rev. C 59, 2487 (1999) [arXiv: nucl-th/9812025].

[30] J. N. Ginocchio, Implication of pseudospin symmetry on relativistic magnetic properties and Gamow-Teller transitions in nuclei. Phys. Rev. C 59, 2487 (1999) [arXiv: nucl-th/9812025].

[31] P. Bicudo, E. Ribeiro, R. Fernandes, The anomalous magnetic moment of quarks. Phys. Rev. C 59, 1107 (1999) [arXiv: hep-ph/9806243].

[32] M. Mekhfi, Tensor charge and anomalous magnetic moment correlation. Phys. Rev. D 72, 114014 (2005) [arXiv: hep-ph/0510059].

[33] G. H. Fuller, Nuclear spins and moments. J. Phys. Chem. Ref. Data 5, 835 (1976).

[34] G. H. Fuller, Nuclear spins and moments. J. Phys. Chem. Ref. Data 5, 835 (1976).

[35] W. Park, A. Park, S. H. Lee, Dibaryons in a constituent quark model. Phys. Rev. D 92, 014037 (2015) [arXiv: nucl-th/1506.01123]. 


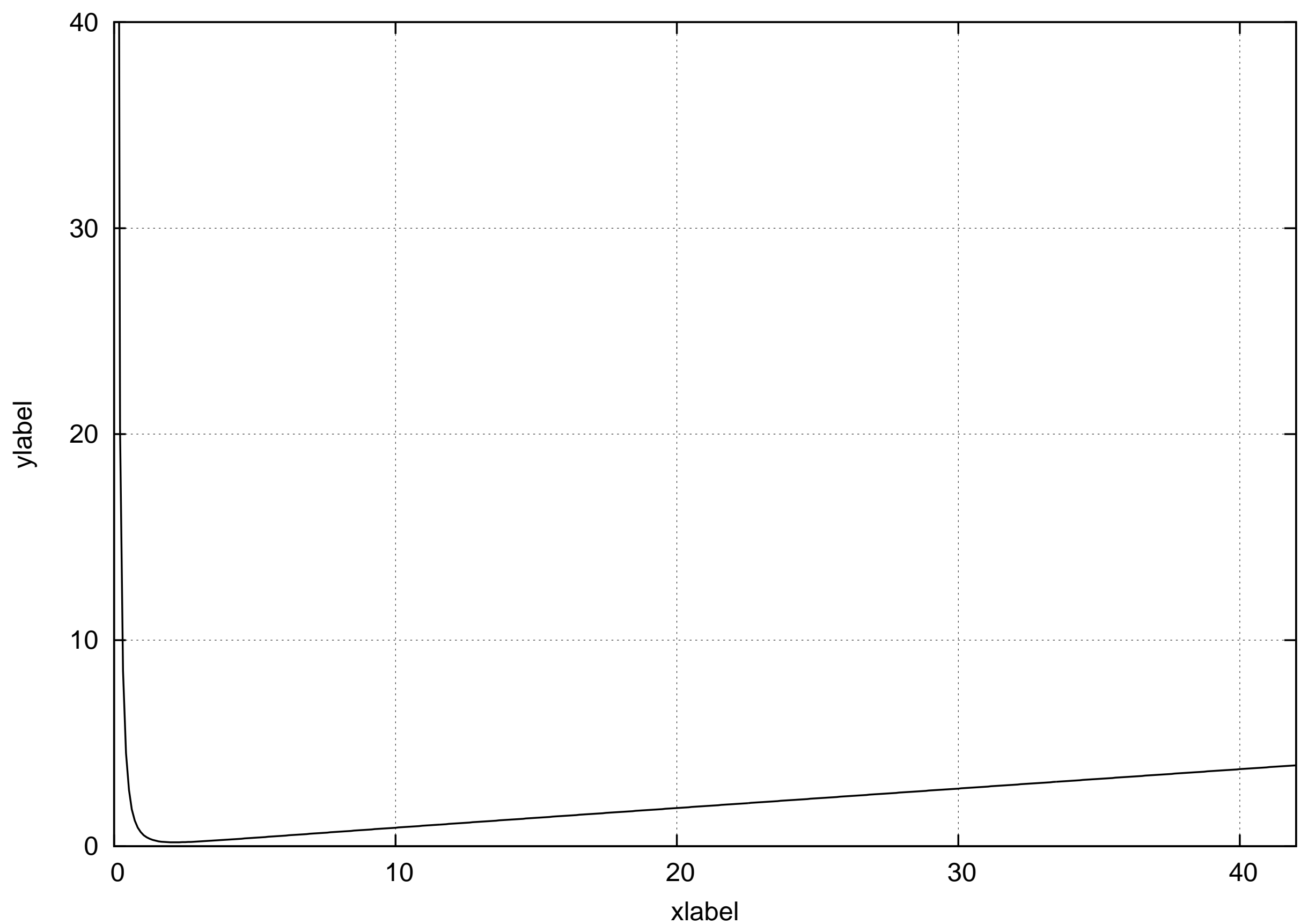

